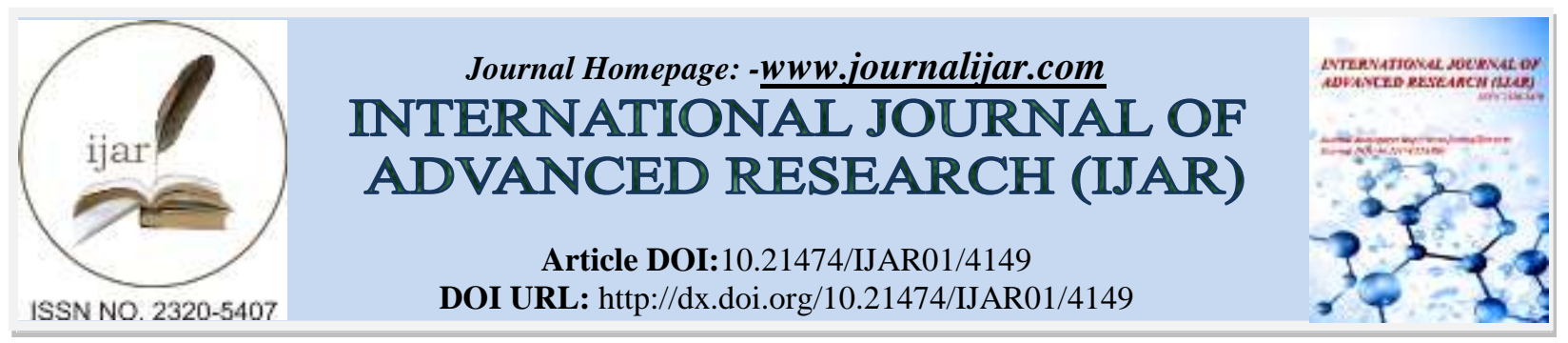

RESEARCH ARTICLE

\title{
A STUDY OF THE TIME VARIATION OF BRANS-DICKE PARAMETER ( $\omega)$
}

\author{
Sudipto Roy. \\ Department of Physics, St. Xavier's College, Kolkata. 30 Mother Teresa Sarani (Park Street), Kolkata - 700016, \\ West Bengal, India.
}

\section{Manuscript Info}

Manuscript History

Received: 05 March 2017

Final Accepted: 06 April 2017

Published: May 2017

Key words:-

Cosmology, Gravitational Constant,

Brans-Dicke theory, Scalar Field,

Accelerated cosmic expansion

\section{Abstract}

The explicit dependence of the dimensionless Brans-Dicke (BD) parameter $(\omega)$ upon time and also upon the equation of state (EoS) parameter $(\gamma)$ has been determined, by solving BD field equations for a space of zero curvature and also the wave equation for the scalar field $(\varphi)$. Empirical expressions of the scale factor and the scalar field have been used for this purpose. Dependence of the present value of the BD parameter $\left(\omega_{0}\right)$ upon a parameter controlling the time variation of the scalar field has been obtained and shown graphically. Time variation of the $\mathrm{BD}$ parameter, for different values of the EoS parameter, characterizing cosmological eras, has been depicted graphically.

Copy Right, IJAR, 2016, All rights reserved.

\section{Introduction:-}

It has been confirmed by high precision observational data and their interpretations that the universe has been expanding with acceleration [1]. This accelerated expansion is said to be caused and controlled by an entity, known as dark energy, whose true nature has not yet been determined. In theoretical calculations, this dark energy is often represented by the cosmological constant $(\Lambda)$, found in General Relativity (GR). Although it accounts for the experimental observations quite well, it has its own limitations [2]. A large number of alternative theoretical models have emerged to explain gravitational observations. The strengths as well as weaknesses of these models can be found in scientific literature [3]. Non-minimally coupled scalar field theories, particularly in the framework of Brans-Dicke (BD) theory, have been found to be highly useful in explaining the phenomenon of accelerated expansion, although they have failed to explain local astronomical experiments. A quintessence scalar field in BD theory has been shown to generate an accelerated expansion of the universe [4]. Introducing a potential term $V(\varphi)$ in BD theory, which is a function of BD scalar field, also allows one to predict an accelerated expansion of the universe [5]. There are major shortcomings of these models. Contrary to the recent observations and theoretical requirements, these models show the matter dominated universe to have an ever accelerating expansion [6,7]. The dimensionless parameter $\omega$ in BD theory plays a very important role in the prediction of observational results. The local astronomical observations are explained very well by GR and demands a high (a few hundreds) value of $\omega$ [8]. In several models in BD theory, the accelerated expansion is found to be generated by a small value of $\omega$, typically of the order of unity. A study has shown that if an interaction of BD scalar field with dark matter is taken into consideration, a generalized BD theory predicts an accelerated expansion even with a high value of $\omega$ [9]. In all these studies, either the BD theory is changed to suit the present requirement or a quintessence scalar field is used to generate sufficient acceleration. Only an interaction between the BD scalar field and the dark matter was used to explain observations in a recent work by Barrow and Clifton [10]. It has also been found that a Brans-Dicke scalar 
field alone can generate an accelerated expansion in the matter dominated era of the universe, without having to consider the presence of any quintessence matter or any interaction between the BD field and the dark matter [11]. A major shortcoming is that these models do not always predict a transition from a phase of deceleration to acceleration. A generalized version of BD theory by Bergman and Wagoner and a more useful form by Nordtvedt can predict this transition $[12,13,14]$. In this generalized theory, the BD parameter $(\omega)$ is regarded as a function of the scalar field $(\varphi)$ and thus it becomes a function of time.

In the present study, we have determined the time dependence of BD parameter which plays a very important role in cosmic expansion. Here we have used BD field equations along with the wave equation for the scalar field. Empirical relations, regarding the time dependence of the scale factor and also the scalar field, have been assumed for this purpose. The choice of the scale factor is such that it would generate a transition of cosmic expansion from a phase of deceleration to acceleration. The constants in this expression have been determined from the experimental observations regarding Hubble parameter and deceleration parameter. The constant $(n)$ in the empirical expression of the scalar field can be estimated from the theoretical restrictions upon the values of $\omega_{0}$ and $\dot{G} / G[11,15]$. This constant determines the rate and nature of change of gravitational constant with time. The time variation of the BD parameter has been shown to depend upon the $\operatorname{EoS}$ parameter $(\gamma)$ of the cosmic fluid, which takes different values for different cosmological eras of the universe. The dependence of the value of $\omega_{0}$ upon the values of other parameters involved in this theoretical formulation has been graphically depicted. The dependence of the BD parameter $(\omega)$ upon time, for a number of choices of different parameter values, has been shown graphically.

\section{Theoretical Model:-}

The gravitational field equations of Brans-Dicke theory, for a universe filled with a perfect fluid and described by FRW space-time with scale factor $a(t)$ and spatial curvature $k$, are given by,

$3 \frac{\dot{a}^{2}+k}{a^{2}}+3 \frac{\dot{a}}{a} \frac{\dot{\varphi}}{\varphi}-\frac{\omega(\varphi)}{2} \frac{\dot{\varphi}^{2}}{\varphi^{2}}=\frac{\rho}{\varphi}$

$2 \frac{\ddot{a}}{a}+\frac{\dot{a}^{2}+k}{a^{2}}+\frac{\omega(\varphi)}{2} \frac{\dot{\varphi}^{2}}{\varphi^{2}}+2 \frac{\dot{a}}{a} \frac{\dot{\varphi}}{\varphi}+\frac{\ddot{\varphi}}{\varphi}=-\frac{P}{\varphi}$

The wave equation for the scalar field $(\varphi)$, in generalized Brans-Dicke theory of gravity, where $\omega$ is a time dependent parameter, is expressed as,

$\ddot{\varphi}+3 \frac{\dot{a} \dot{\varphi}}{a}=\frac{\rho-3 P}{2 \omega+3}-\frac{\dot{\omega} \dot{\varphi}}{2 \omega+3}$

The energy conservation for the cosmic fluid is written as,

$\dot{\rho}+3 \frac{\dot{a}}{a}(\rho+P)=0$

The equation of state of the fluid is expressed as,

$P=\gamma \rho$

Here $\gamma$ is the equation of state $(\mathrm{EoS})$ parameter for the cosmic fluid, having different values for different cosmological eras. The values of $\gamma$ are -1 (vacuum energy dominated era), 0 (matter dominated era), $1 / 3$ (radiation dominated era), 1 (massless scalar field dominated era).

The solution of equation (4), using equation (5), is obtained as,

$\rho=\rho_{0} a^{-3(1+\gamma)}$

Following empirical expressions, for the scale factor $(a)$ and the scalar field $(\varphi)$, have been used for the present study.

$a=a_{0} \operatorname{Exp}\left[-\alpha t_{0}^{\beta}\right] \operatorname{Exp}\left[\alpha t^{\beta}\right]$

$\varphi=\varphi_{0}\left(a / a_{0}\right)^{n}$ 


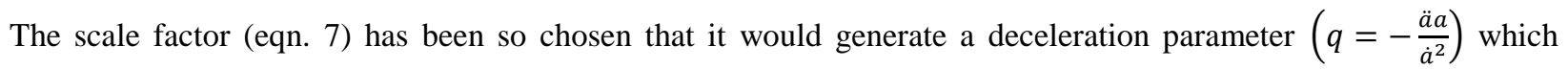
changes its sign with time. This signature flip of the deceleration parameter has been reported in several studies, indicating a transition of the universe from a phase of decelerated expansion to an accelerated one [14]. Here $\alpha, \beta$ should have the same sign to ensure the increase of scale factor with time (in an expanding universe). The empirical expression of the scalar field $\varphi$ (eqn. 8) has been obtained from a study by Banerjee and Ganguly in 2009 [14].

For $0<\beta<1$ and $\alpha>0$ one finds that, $q>0$ for $t=0$ and $q \rightarrow-1$ as $t \rightarrow \infty$, showing a signature flip of $q$ clearly.

Using this scale factor one obtains,

$H=\frac{\dot{a}}{a}=\alpha \beta t^{(\beta-1)}$

$q=-\frac{\ddot{a} a}{\dot{a}^{2}}=-1+\frac{1-\beta}{\alpha \beta} t^{-\beta}$

To determine the values of the constants $\alpha$ and $\beta$ we apply the following conditions:

1) at $t=t_{0}$ we have $H=H_{0}$

2) at $t=t_{0}$ we have $q=q_{0}$

Applying these conditions we get,

$\beta=1-H_{0} t_{0}\left(1+q_{0}\right)$

$\alpha=\frac{H_{0}}{1-H_{0} t_{0}\left(1+q_{0}\right)} t_{0}\left\{H_{0} t_{0}\left(1+q_{0}\right)\right\}$

Using equations (7) and (8) in (1) and taking $k=0$, one obtains the following BD parameter $\left(\omega_{1}\right)$.

$\omega_{1}=\frac{2}{n^{2}}\left(3+3 n-\frac{\rho}{\varphi H^{2}}\right)$

Using equations (7) and (8) in (2) and taking $k=0$, one obtains the following BD parameter $\left(\omega_{2}\right)$.

$\omega_{2}=\frac{2}{n^{2}}\left(2 q-1-n+n q-n^{2}-\frac{\gamma \rho}{\varphi H^{2}}\right)$

A weighted average of $\omega$, using (13) and (14), can be expressed as,

$\omega=\frac{x \omega_{1}+y \omega_{2}}{x+y}$

Here $x$ and $y$ are the weight parameters for $\omega_{1}$ and $\omega_{2}$ respectively and they can be arbitrarily varied in the final expression of $\omega$ (eqn. 18) to determine their effect on the time dependence of $\omega$.

Using equations (13) and (14), in equation (15), one obtains,

$\omega=\frac{x\{3(1+n)-z\}+y\{(n+2) q-n(1+n)-1-\gamma z\}}{(x+y) n^{2} / 2}$

where, $z=\frac{\rho}{\varphi H^{2}}=\frac{\rho_{0} a_{0}{ }^{-m} t^{2(1-\beta)} \operatorname{Exp}\left[-m \alpha\left(t^{\beta}-t_{0}{ }^{\beta}\right)\right]}{\alpha^{2} \beta^{2} \varphi_{0}}$

with $m=3+3 \gamma+n$

and $q=-1+\frac{1-\beta}{\alpha \beta} t^{-\beta}$

Using equation (16) one gets,

$\dot{\omega} \equiv \frac{d \omega}{d t}=\frac{-x \dot{z}+y\{(n+2) \dot{q}-\gamma \dot{z}\}}{(x+y) n^{2} / 2}$ 
or,

$\dot{\omega}=\frac{2}{n^{2}(x+y)}\left[\frac{y(n+2)(\beta-1) t^{-(\beta+1)}}{\alpha}-\frac{(x+y \gamma) \rho_{0}\left[2-\beta\left(2+m \alpha t^{\beta}\right)\right] a^{-m}}{\alpha^{2} \beta^{2} \varphi_{0}}\right]$

with $m=3+3 \gamma+n$

From equation (3) one gets the following expression for $\omega(t)$,

$\omega(t)=\frac{1}{2} \frac{\rho(1-3 \gamma)-\dot{\omega} \dot{\varphi}}{\ddot{\varphi}+3(\dot{a} \dot{\varphi}) / a}-\frac{3}{2}$

where,

$\rho=\rho_{0} a_{0}^{-3(1+\gamma)} \operatorname{Exp}\left[-3 \alpha(1+\gamma)\left(t^{\beta}-t_{0}{ }^{\beta}\right)\right]$

$\dot{a}=\alpha \beta t^{\beta-1} a$

$\dot{\varphi}=n \alpha \beta t^{\beta-1} \varphi$

$\ddot{\varphi}=n \alpha \beta t^{\beta-2}\left(\beta+\alpha \beta n t^{\beta}-1\right) \varphi$

$\dot{\omega}=\frac{2 y(n+2)(\beta-1) t^{-(\beta+1)}}{n^{2}(x+y) \alpha}-\frac{2(x+y \gamma) \rho_{0}\left[2-\beta\left(2+m \alpha t^{\beta}\right)\right] a^{-m}}{\alpha^{2} \beta^{2} \varphi_{0} n^{2}(x+y)}$

with $m=3+3 \gamma+n$

Thus equation (18) represents an expression of the BD parameter, showing its explicit time dependence, as well as its dependence upon the EoS parameter $(\gamma)$ and other relevant cosmological parameters.

According to BD theory, $G=1 / \varphi$. Combining this relation with equation (8) one gets,

$n=-\frac{1}{H_{0}}\left(\frac{\dot{G}}{G}\right)_{t=t_{0}}$

Using equation (19), one may determine the value of the parameter $n$ from the experimental values of $H_{0}$ and $\left(\frac{\dot{G}}{G}\right)_{t=t_{0}}$. This parameter controls the rate of change of $\varphi(\equiv 1 / G)$ with time. The experimental observations regarding $\left(\frac{\dot{G}}{G}\right)_{t=t_{0}}$ has been reported by many researchers where it has been shown to be both positive and negative [16]. The value of $n$ should be so chosen that $\left|\left(\frac{\dot{G}}{G}\right)_{t=t_{0}}\right| \leq 4 \times 10^{-10} \mathrm{Yr}^{-1}$ (Weinberg, 1972) [15]. Therefore, using equation (19), one may express this requirement as $|n| \leq 5.445$ taking $H_{0}=7.348 \times 10^{-11} \mathrm{Yr}^{-1}$.

According to an advanced study by Banerjee and Pavon, the present value of BD parameter $\left(\omega_{0}\right)$ must have the following range of variation $[11,18]$.

$-3 / 2<\omega_{0}<0$

Using equations (18) and (20), one obtains,

$0<\left[\frac{\rho(1-3 \gamma)-\dot{\omega} \dot{\varphi}}{\ddot{\varphi}+3(\dot{a} \dot{\varphi}) / a}\right]_{t=t_{0}}<3$

Using equations (6) and (18), it can be shown that,

$\frac{\partial \omega}{\partial \gamma}=\frac{1}{2} \frac{-3 \rho[(1-3 \gamma) \ln a+1]-\dot{\varphi}(\partial \dot{\omega} / \partial \gamma)}{\ddot{\varphi}+3(\dot{a} \dot{\varphi}) / a}$ 
Using the fact that $\gamma$ decreases with time (from the sequence of cosmological eras) and $\rho$ also decreases (from eqn. 6), equation (22) allows one to determine the effect of change of $t$ and $\gamma$ upon the value of $\frac{\partial \omega}{\partial \gamma}$.

The values of different cosmological parameters used for the present study are given below.

$H_{0}=\frac{72 \frac{K m}{s}}{M p c}=2.33 \times 10^{-18} \mathrm{sec}^{-1}, q_{0}=-0.55, \rho_{0}=2.83 \times 10^{-27} \mathrm{Kg} \mathrm{m}^{-3}$

$\varphi_{0}=\frac{1}{G_{0}}=1.498 \times 10^{10} \mathrm{Kg}^{2} \mathrm{~m}^{-2} \mathrm{~N}^{-1}, t_{0}=4.36 \times 10^{17} \mathrm{~s}$

\section{Results:-}

Figures 1 and 2 show the variation of $\omega_{0}$ as a function of $n$ for different combinations of values of the parameters $x$ and $y$. These graphs clearly show the ranges of $n$ for which equation (20) is satisfied, for different combinations of $x$ and $y$. In Figure 2, most of the values of $\omega_{0}$ are in the negative region. The dotted lines in these two figures indicate the level of $\omega_{0}=-3 / 2$.

Figures 3, 4, 5 and 6 show the variation of the BD parameter $(\omega)$ as a function of time, for $n=-5$, corresponding to four values of the EoS parameter $\left(\gamma=1, \frac{1}{3}, 0,-1\right)$ respectively. Figures 7, 8, 9 and 10 show the variation of the BD $(\omega)$ parameter as a function of time, for $n=+3$, corresponding to four values of the EoS parameter $\left(\gamma=1, \frac{1}{3}, 0,-1\right)$ respectively. Figures 5, 9 show the time variation of the BD parameter for the matter dominated universe with negligible pressure $(\gamma=0)$. The red curves in both figures satisfy the criterion of equation (20), and thereby expected to represent the correct time dependence of $\omega$. In these two figures we find $\omega$ to become more and more negative with time, at a gradually decreasing rate. Figures 1 and 2 show that the negative values of $n$ are more consistent with the requirement expressed by equation (20). According to equations (8) and (19), a negative value of $n$ means a decrease of scalar field $(\varphi)$ with time or equivalently an increase in gravitational constant with time. There are several studies that show a decrease of scalar field with time [14, 17-20].

\section{Conclusions:-}

Using Brans-Dicke field equations for flat space, we have determined the time dependence of the BD parameter $(\omega)$ for different cosmological eras, characterized by different values of the EoS parameter $(\gamma)$. An empirical scale factor (eqn. 7) has been used for this purpose. One may think of a better method of study through the determination of the scale factor by solving the field equations, using the empirical expression of the scalar field $(\varphi)$. According to a study by Banerjee and Pavon [11], the present value of $\omega$ is supposed to be negative and it should also be greater than $-3 / 2$. This requirement has been shown to be fulfilled if the gravitational constant increases with time (i.e. for the negative values of $n$ ). To obtain the correct combination of the parameters, $x$ and $y$, one must take into account equation (20) and also the requirement that $|n| \leq 5.445$, based on the criterion of $\left|\left(\frac{\dot{G}}{G}\right)_{t=t_{0}}\right| \leq 4 \times 10^{-10} \operatorname{Yr}^{-1}$, obtained from a study by S. Weinberg [15]. The present formulation can be regarded as an improvement over some previous studies [17, 18], where the empirical relation for the scale factor had a power-law dependence upon time. It generates a time independent deceleration parameter which is not at all acceptable as per the recent studies in this regard [14]. 


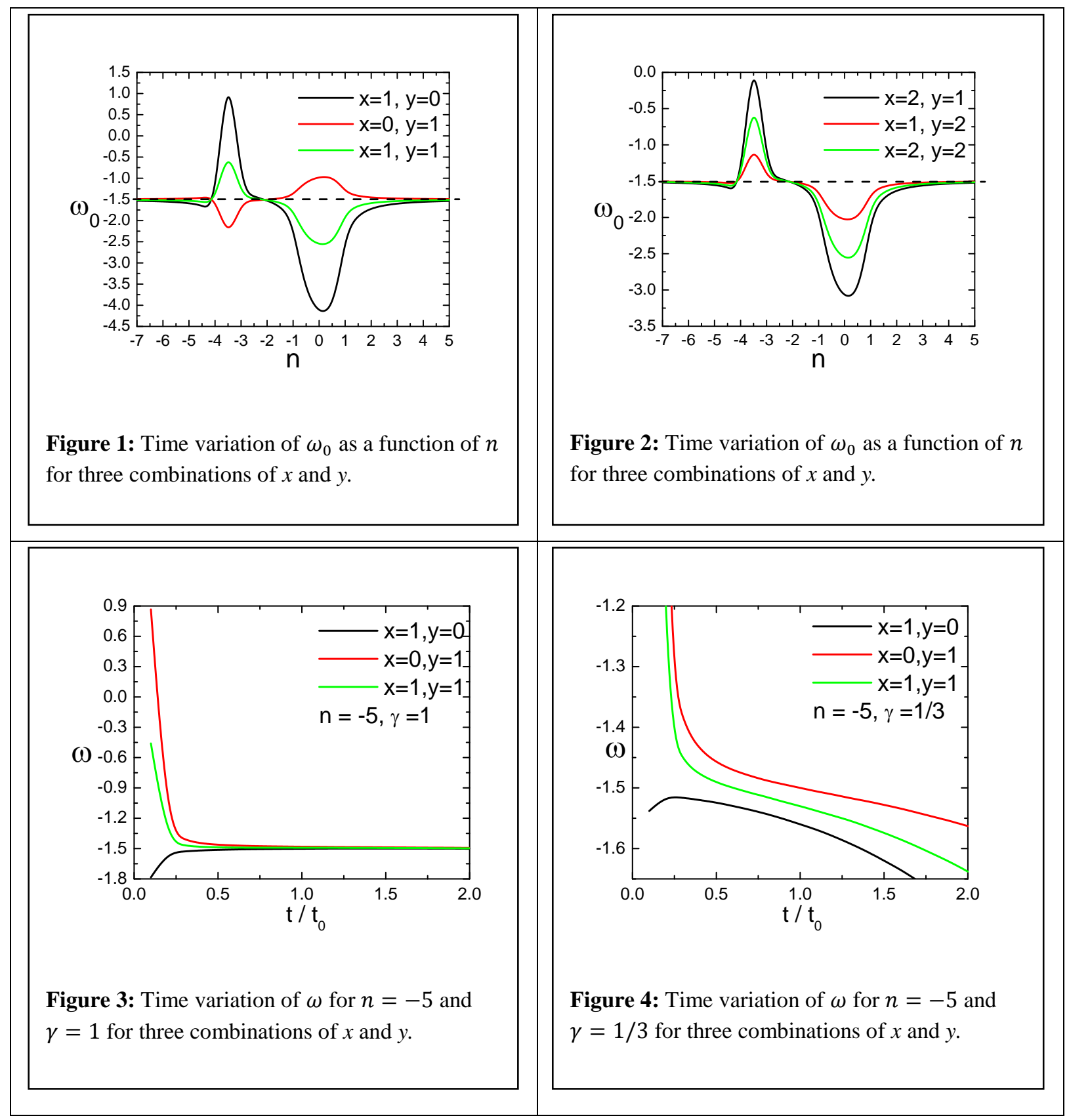




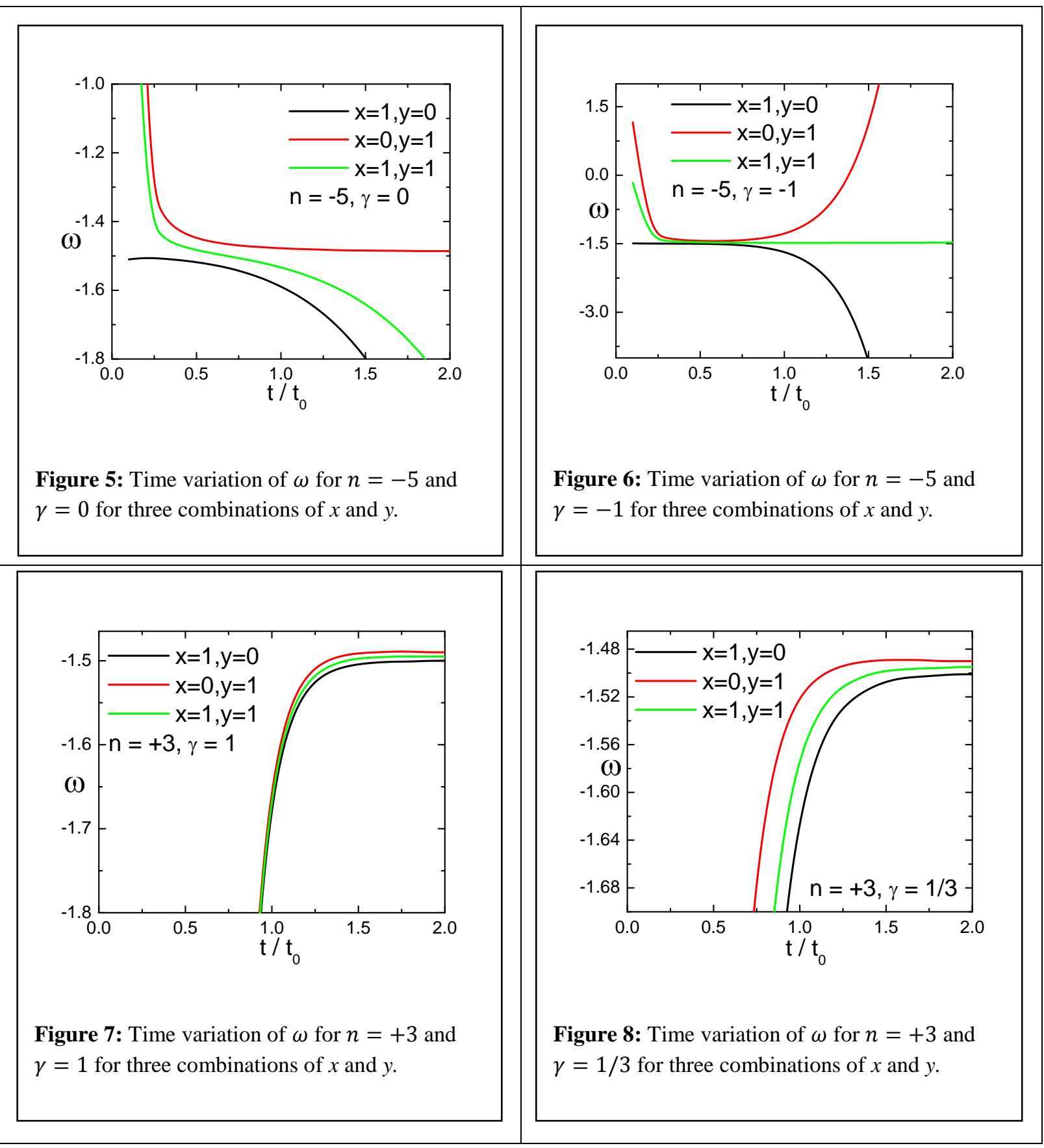




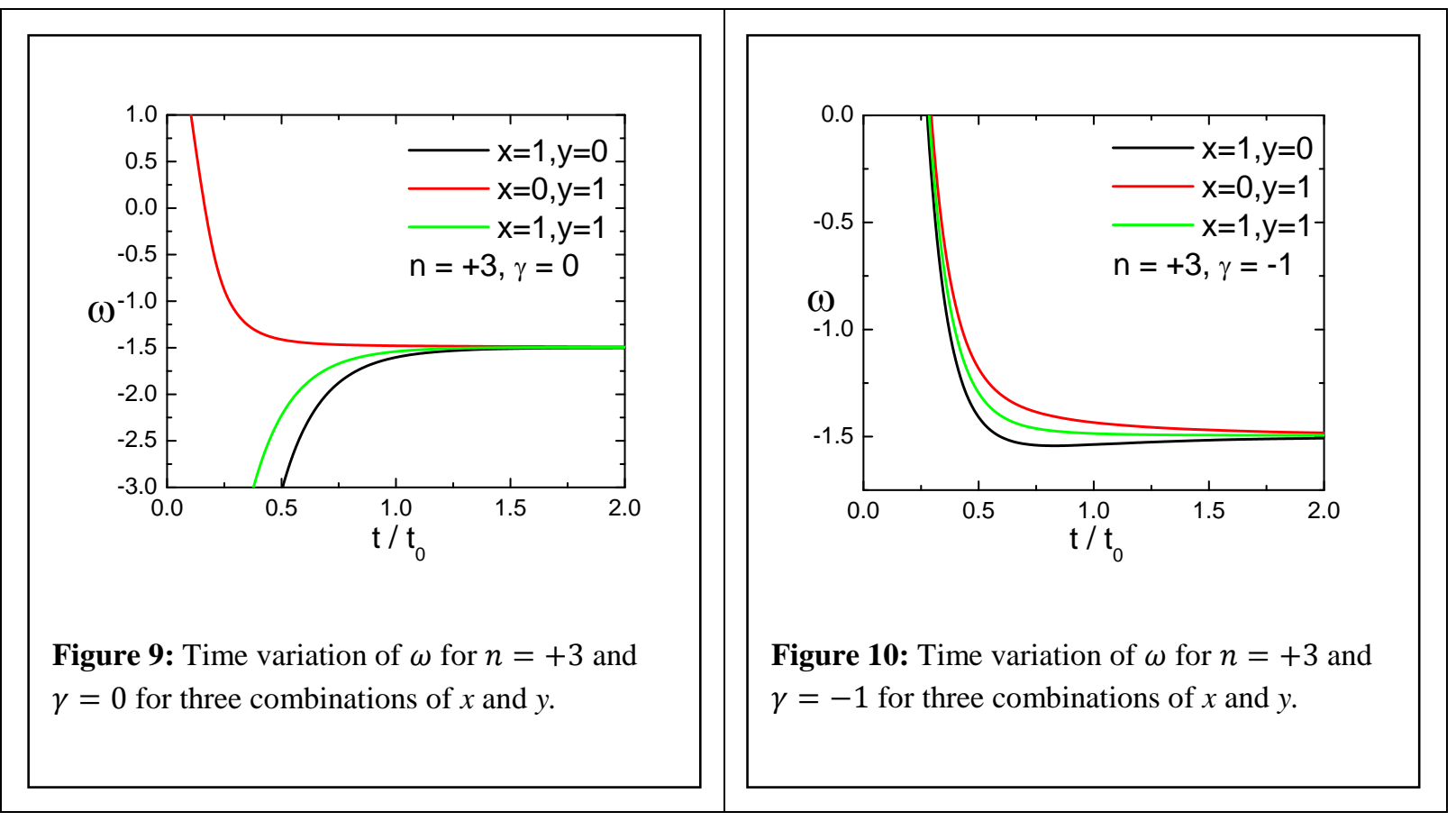

\section{References:-}

1. Bennet, C.L. et al. (2003): First-Year Wilkinson Microwave Anisotropy Probe (WMAP) Observations: Preliminary maps and basic results. The Astrophysical Journal Supplement Series, 148(1): 1-27.

2. Sahni, V. and Starobinsky, A. (2000): The case for a positive cosmological $\Lambda$ term. Int. J. Mod. Phys. D, 09(4): 373-443.

3. Padmanabhan, T. (2003): Cosmological Constant-the weight of the vacuum. Phys. Rept., 380: $235-320$.

4. Sen, A.A., Sen, S. (2001): Cosmology in scalar tensor theory and asymptotically de-Sitter Universe. Mod. Phys. Lett. A, 16:1303-1314.

5. Sen, S. and Seshadri, T.R. (2003): Self interacting Brans Dicke Cosmology and Quintessence. Int.J.Mod.Phys. D, 12: 445-460.

6. Riess, A.G. et al. (2001): The farthest known supernova: support for an accelerating universe and a glimpse of the epoch of deceleration, Astrophys. J., 560: 49-71.

7. Padmanabhan, T. and Roy Choudhury, T. (2003): A theoretician's analysis of the supernova data and the limitations in determining the nature of dark energy. Mon. Not. R. Astron. Soc., 344: 823-834.

8. Will, C.M., (1993): Theory and Experiment in Gravitational Physics, Cambridge University Press, Chapter-5, pp. 123-126.

9. Banerjee, N. and Das, S. (2006): An interacting scalar field and the recent cosmic acceleration. Gen. Relativ. Gravit., 38 (5): 785-794.

10. Clifton, T. and Barrow, J.D. (2006): Decaying Gravity. arXiv:gr-qc/0603116v2: 1-13.

11. Banerjee, N. and Pavon, D. (2001): Cosmic acceleration without quintessence. Phys. Rev. D, 63: 043504043508.

12. Bergman, P.G. (1968): Comments on the scalar-tensor theory. Int. J. Theor. Phys., 1(1): 25-36.

13. Nordtvedt, K. (1970): Post-Newtonian metric for a general class of scalar-tensor gravitational theories and observational consequences. Astrophys. J., 161: 1059-1067.

14. Banerjee, N. and Ganguly, K. (2009): Generalised Scalar-Tensor theory and the cosmicacceleration. Int. J. Mod. Phys. D, 18 (3): 445-451.

15. Weinberg, S. (1972):Gravitation and Cosmology: Principles and Applications of the General Theory of Relativity. John Wiley \& Sons (Asia) Pte. Ltd., Singapore, pp. 630.

16. Ray, S., Mukhopadhyay, U. and Dutta Choudhury, S.B. (2007): Dark energy models with time-dependent gravitational constant. Int. J. Mod. Phys. D, 16: 1791 - 1802. 
17. Jamil, M. and Momeni, D. (2011): Evolution of the Brans-Dicke Parameter in Generalized Chameleon Cosmology. Chin. Phys. Lett., 28 (9), 099801: 1-4.

18. Sahoo, B.K. and Singh, L.P. (2002): Time Dependence of Brans-Dicke Parameter $\omega$ for an Expanding Universe. Mod. Phys. Lett. A, 17 (36): 2409-2415.

19. Roy, S. (2016): Time Evolution of Various Cosmological Parameters and Their Inter- Dependence in the Framework of Brans-Dicke Theory. IOSR Journal of Mathematics, 12 (3), Version VII: 27-35.

20. Roy, S. (2016): A Theoretical Study of the Cosmic Expansion in the Framework of Brans-Dicke Theory. IOSR Journal of Applied Physics, 8 (3), Version III: 04-12. 